\title{
Volumetric and hygrometric performance of the NT8®, an advanced liquid handler for high throughput crystallization screening
}

\author{
Aggarwal, Mayank; Damrau, William; Rodionov, Dmitry \\ Email Contact: lance.ramsey@formulatrix.com
}

The formation of the Structural Genomics Consortium in 2004 has significantly increased the number of structures deposited to Protein Data Bank (PDB) per year for two reasons: the advent of a large group of researchers focused on solving and depositing structures and the creation of the automation space for crystallization screening. It would not be possible without automated imagers as routine inspection of crystallization experiments is very time-consuming. However, specialized liquid handlers capable of dispensing sub-microliter volumes were, and remain, the absolute requirement for accelerating crystallization screening.

These liquid handlers are frequently called dropsetters and allow researchers to set up the experiments faster, but more importantly, to set up more screens with the same amount of sample due to the reliable low-volume performance of the instruments. Unfortunately, lower volume crystallization experiments are very sensitive to evaporation, which can be countered by either the instrument speed, which can not be increased without other drawbacks, or by the addition of humidity control.

The NT8 is one of the most recent automation solutions to join the ranks of the dropsetters. The system features a modular head construction, allowing for a combination of Low Volume (LV) (=<1.5 uL), High Volume (HV) (20-200 uL), and Lipidic Cubic Phase (LCP) (as low as $0.03 \mathrm{uL}$ ) sub-assemblies. The LV head uses proprietary tips that can be automatically washed and reused to reduce the consumable cost to the researcher and environmental impact. In contrast, the other two heads use commercially-available consumables. The NT8 also includes a built-in LCP mixer and the most advanced humidity control with proportional response to prevent evaporation.

We have previously reported the impact of humidity level on evaporation in crystallization experiments. Here we present the NT8's liquid handling performance highlighting the single-digit relative inaccuracy and imprecision across the typical usage scenarios. We also examine the NT8's speed at reaching the target humidity level as well as the ability to maintain the setpoint. 